\title{
Diferencias socioterritoriales en el conocimiento y uso de dispositivos ecológicos para la vivienda de la Ciudad de México
}

\section{Socio-territorial differences in the knowledge and use of ecological devices for housing in Mexico City}

Perla Yannelli Fernández Silva*

David Morillón Gálvez**

\section{Resumen}

La importancia de incrementar el número de viviendas sustentables, y con ello contribuir a frenar los efectos del cambio climático, responde a que $4.62 \%$ de las emisiones de $\mathrm{CO}_{2}$ provienen del sector habitacional. Las ecotecnologías para la vivienda son dispositivos con potencial para reducir emisiones de gases de efecto invernadero, así como para ahorrar. Estos instrumentos están cada vez más disponibles en el mercado, sin que se utilicen de manera generalizada en las viviendas de la Ciudad de México. Este trabajo identifica las diferencias entre la población que conoce y utiliza ecotecnias en las viviendas de la capital mexicana. Por medio de encuestas y estadísticas se encontraron diferencias significativas entre cinco estratos socioterritoriales en las formas de conocimiento y uso de ecotecnologías para la vivienda.

* Universidad Cuauhtémoc de Querétaro, Maestría en Arquitectura Sustentable. Dirección: Prol. Bernardo Quintana A. 229, Loma Dorada, 76060, Querétaro, México. Correo: pefernandez@ucq.edu.mx ORCID: https://orcid.org/0000-0001-6867-8318

** Universidad Nacional Autónoma de México, Instituto de Ingeniería. Dirección: Edificio 12, Ciudad Universitaria, 04510, Ciudad de México. Correo: damg@ pumas.ii.unam.mx ORCID: https://orcid.org/0000-0002-9178-3092 
Palabras clave: ecotecnologías para la vivienda; análisis socioterritorial; construccionismo social; Ciudad de México.

\section{Abstract}

Increasing the number of sustainable housing contributes to decreasing the effects of climate change, due to $4.62 \%$ of the $\mathrm{CO}_{2}$ emissions that come from the housing sector. This work identifies the socioeconomic and territorial factors of the users who know and use the eco-technologies for housing differentially. Through surveys and significant differences in statistics, we found the ways in which each socioeconomic group knows and uses the ecological devices for existing housing in Mexico City.

Keywords: eco-technologies for housing, socio-territorial analysis, social constructionism, Mexico City.

\section{Introducción}

En las últimas cinco décadas, el tema de la sustentabilidad ha cobrado relevancia cada vez con mayor intensidad (Meadows, Randers y Behrens, 1972) debido al incremento de la contaminación ambiental y a sus efectos asociados con el cambio climático. Simultáneamente, el desarrollo tecnológico ha buscado cambiar la relación ser humano-naturaleza al modificar las formas tradicionales de extracción y transformación de los recursos naturales desde diferentes perspectivas y modelos; ello aunado a la búsqueda de justicia social.

Bajo esta perspectiva es que las ecotecnologías se presentan como un instrumento cada vez más difundido para equilibrar la relación entre el ser humano y la naturaleza mediante un paradigma global y complejo. Las formas de definir a las ecotecnologías varían dependiendo de la visión y la disciplina que las conceptúan; por ejemplo, la ingeniería ecológica y la ecología industrial permean la literatura anglosajona, mientras que las referencias científicas en español aún son escasas y por lo general se refieren a tecnologías alternativas, dispositivos eficientes para el uso de agua y energía, así como a algunas aplicaciones arquitectónicas (Ortiz, Masera y Fuentes, 2014). 
Entre las diferentes modalidades que se le da a las ecotecnologías, ${ }^{1}$ están las que se aplican en la edificación, específicamente a viviendas tipificadas como sustentables, ecológicas y verdes, y que se refieren a espacios habitacionales con bajas emisiones de gases de efecto invernadero, bajo consumo de agua potable, eficiencia en el manejo de residuos, uso de sistemas de confort bioclimático, así como uso de materiales locales; adicionalmente, dichas viviendas ofrecen ventajas sociales y económicas para garantizar la calidad de vida de sus ocupantes, ${ }^{2}$ tanto en zonas rurales como urbanas.

El objetivo de esta investigación es identificar los principales aspectos socioeconómicos de quienes conocen y usan en mayor o menor grado las ecotecnologías para la vivienda existente en la Ciudad de México. Este trabajo se centra en el análisis de los grupos de sujetos que potencialmente podrían impulsar la transición hacia la sustentabilidad. Si bien el enfoque no se dirige a la evaluación de las viviendas y las ecotecnologías respecto a la eficiencia en las emisiones de $\mathrm{CO}_{2}$, ni a la calidad de vida de los usuarios, sí se intenta delinear el perfil socioeconómico de la población cuyo conocimiento y convencimiento del uso de ecotecnologías es mayor. Se identifican los factores socioeconómicos y territoriales que condicionan la forma de conocimiento y uso de las ecotecnologías para la vivienda, por ejemplo: el nivel socioeconómico, la escolaridad, la edad de la población, el acceso a la información, la experiencia de los usuarios, el tipo de vivienda y su localización, principalmente. La pregunta que guía el trabajo es, ¿cómo cambia el conocimiento

${ }^{1}$ Ecotecnologías, entendido en un sentido amplio, mientras que las ecotecnias se refieren a las aplicaciones prácticas de éstas.

${ }^{2}$ Adicionalmente, ONU-Hábitat (2019) considera que una vivienda sostenible no es sólo "una unidad o un grupo de edificios verdes autosostenibles, sino una práctica residencial, amigable con el ambiente y con la sociedad que se integra con los sistemas urbanos y/o asentamientos [...] también significa privacidad, espacio adecuado, accesibilidad física, seguridad apropiada, seguridad de tenencia, estabilidad y confiablidad estructural, iluminación, ventilación y calefacción apropiadas; también implica infraestructura básica adecuada, como suministro de agua y servicios sanitarios y de manejo de residuos; cualidades ambientales adecuadas y factores relacionados con la salud; así como una buena ubicación respecto a las instalaciones básicas y laborales. Adicionalmente, todos los elementos anteriores deben ser asequibles". 
y uso de ecotecnologías en las viviendas existentes en la Ciudad de México a partir de las diferencias socioeconómicas de los usuarios?

Para lograr este objetivo, se realizó un estudio transversal cuantitativo y correlacional sobre las ecotecnologías instaladas en viviendas existentes en cinco estratos socioterritoriales de la Ciudad de México, tanto en la porción urbana como en la rural. En este trabajo, las unidades de análisis no son las viviendas, sino sus usuarios como agentes que, de manera diferenciada, conocen, deciden, operan e incorporan o no las ecotecnologías a su vida cotidiana.

\section{Ecotecnologías para la vivienda en México. Antecedentes}

La vivienda tradicional o vernácula de México históricamente se ha caracterizado por cumplir con estándares óptimos de confort bioclimático y de integración con el entorno (Morillón, 2009), aunque en la época moderna del siglo XX, con la industrialización de los materiales y procesos constructivos, se diluye el estrecho vínculo entre la casa y el medio ambiente. Es hasta las últimas tres décadas del siglo $\mathrm{XX}$, bajo el discurso de la sustentabilidad (Lezama y Domínguez, 2006), cuando en México se retoma de manera paulatina el proceso de integración entre la arquitectura y el medio ambiente a través de las ecotecnologías. Son los trabajos pioneros de Jesús Arias Chávez (Instituto Politécnico Nacional), José Luis Alcalá y Enrique Flores (Universidad de Guadalajara) y Fritche y Zapata (en San Luis Potosí) (Morillón, 2011), los que se reconocen como las primeras propuestas de viviendas sustentables con uso de ecotecnologías y diseño bioclimático. Adicionalmente, existen otras investigaciones que, desde finales del siglo XX, abonaron al desarrollo de ecotecnologías, como son las de Armando Deffis Caso (1982), Fernando Tudela (1982) y del Centro de Ecodesarrollo (Portillo y Sirvent, 1987), entre otras.

Aquí es fundamental plantear dos vertientes que coexisten respecto a las ecotecnologías para la vivienda:

1) Las tecnificadas, elaboradas industrialmente y que cumplen con criterios de normas oficiales mexicanas y estándares internacionales (por ejemplo, celdas fotovoltaicas, calentadores solares, 
dispositivos ahorradores de agua, boiler de paso, películas y aislantes térmicos, entre otras).

2) Las no tecnificadas, de elaboración casera con uso de materiales económicos o disponibles localmente (como sistemas de captación de agua de lluvia, baños secos, composta, estufas solares, entre otras).

Una clasificación complementaria a ésta, es la que proponen Morillón y Morillón (1993) para catalogar sistemas pasivos, semipasivos o activos. ${ }^{3}$ Por lo tanto, las ecotecnologías no deben ser consideradas sólo las constructivas arquitectónicas que están disponibles en el mercado, sino también incluir aquellas que permiten a los usuarios incorporar soluciones a su vivienda a través del ahorro, ordenamiento, reciclaje o recolección de recursos.

En correspondencia, la definición de ecotecnologías para la vivienda puede plantearse desde acepciones distintas; en este caso se exponen las posturas liberal mercantilista, culturalista e institucional. En la primera de ellas se argumenta que las ecotecnologías corresponden a

[...] la utilización de prácticas y materiales respetuosos del medio ambiente (con ventaja ambiental o ambientalmente preferibles) en la planeación, diseño, ubicación, construcción, operación y demolición de edificaciones [CCA, 2008].

Esta definición tiene sus raíces en el sistema de construcción anglosajón de vivienda, que por lo general se desarrolla en sectores sociales altos, así como en edificaciones corporativas con elevados estándares de calidad definidos por consejos de construcción especializados.

Para el caso de la visión culturalista, Ortiz, Masera y Fuentes definen las ecotecnologías como:

${ }^{3}$ Los sistemas activos son aquellos cuyo desempeño depende de su diseño y componentes internos, por su equipamiento mecánico o eléctrico; en los pasivos, el desempeño del equipo requiere de la interacción de los usuarios para completar su proceso de funcionamiento; por último, los semipasivos están determinados porque su desempeño es aleatorio, en ocasiones activos, y otras veces pasivo (Morillón y Morillón, 1993). 
[...] los dispositivos, métodos y procesos que propician una relación armónica con el ambiente y buscan brindar beneficios sociales y económicos tangibles a sus usuarios, con referencia a un contexto socioecológico específico [Ortíz, Masera y Fuentes, 2014].

Por último, la definición institucionalizada concibe a las ecotecnologías como:

[...] sistemas y productos que utilizan avances tecnológicos con objeto de optimizar el uso del agua, la energía y el gas en el interior de una vivienda, así como productos o sistemas que aprovechan los recursos renovables inherentes a su ubicación [Conavi, 2018].

En todos los casos, las ventajas generales que se derivan del uso de las ecotecnologías en la edificación son: 1) reducción de la demanda de combustibles fósiles y de la emisión de gases de efecto invernadero (GEI); 2) prevención del agotamiento de los recursos naturales; 3) eliminación de residuos que provocan la contaminación del aire, agua y suelo; 4) beneficios asociados con la salud humana; 5) confort climático; 6) bienestar y calidad de vida; y 7) ahorro monetario. Las ecotecnologías para la vivienda se vuelven imprescindibles para minimizar los efectos del calentamiento global cuando las cifras muestran que el $17 \%$ de la energía final en México es utilizada por el sector residencial (Sener, 2011); entre 30 y 40\% del agua potable en México es para uso doméstico (Carabias y Landa, 2005); y se producen diariamente $5.85 \mathrm{~kg}$ de desechos sólidos por vivienda (Rojas, 2015). Por lo tanto, $4.62 \%$ de las emisiones de $\mathrm{CO}_{2}$ provienen del sector habitacional ${ }^{4}$ (INEGYCEI, 2014).

Con todo lo anterior, es importante distinguir lo que se ha hecho, los logros, y lo que falta por hacer para que las ecotecnologías para la vivienda sean utilizadas de forma generalizada, no sólo en la Ciudad de México, sino en todo el país.

En cuanto a lo que se ha hecho a través de la política nacional de vivienda, en el sexenio de 2012 a 2018 la mayoría del esfuerzo se enfocó a que la producción de vivienda social nueva incorporara

\footnotetext{
${ }^{4}$ https://www.gob.mx/cms/uploads/attachment/file/312045/INEGYCEI6CN_ 26_marzo_2018.pdf
} 
cada vez un mayor número de ecotecnologías; para ello se diseñaron iniciativas y programas interinstitucionales promovidos por el Gobierno Federal, con la participación de instancias internacionales como el Banco Interamericano de Desarrollo, la Agencia de Cooperación Alemania-México (GIZ), el Banco Mundial, el Banco de Desarrollo Alemán (KfW) y ONU-Hábitat; además de la colaboración de instituciones académicas, inmobiliarias, constructoras y asociaciones civiles, que también contribuyeron a transitar hacia modelos de edificación de vivienda sustentable. Dentro de las acciones que el Gobierno Federal ha implementado ${ }^{5}$ se encuentran el Sistema de Evaluación de la Vivienda Verde (Sisevive-Ecocasa), ${ }^{6}$ operado por la Comisión Nacional de Vivienda (Conavi) y la Hipoteca Verde del Infonavit, así como la NAMA ${ }^{7}$ de Vivienda, en las modalidades EcoCasa NAMA Facility \& LAIF, bajo el auspicio de la Sociedad Hipotecaria Federal (SHF). Es importante recalcar que el programa Sisevive se diseñó para ser dirigido por la iniciativa privada a través de las empresas desarrolladoras de vivienda, en operación con los programas de créditos y subsidios federales.

Adicionalmente, grupos colegiados coordinados por la Comisión Nacional de Uso Eficiente de la Energía (Conuee) trabajan en la definición y actualización de las normas oficiales mexicanas para la eficiencia energética, así como en el fomento a la certificación de productos, procesos y servicios vinculados con el sector vivienda.

En lo referente a los resultados, ya sea por la implementación de programas o por los propios procesos de adopción de las ecotecnologías, se sabe que en el periodo de 2015 a 2018 se certificaron 246795 viviendas a nivel nacional, bajo el sello Sistema de Evaluación de la Vivienda Verde (Sisevive), ${ }^{8}$ herramienta diseñada para certificar la

${ }^{5}$ Alineadas normativamente con el Plan Nacional de Desarrollo, 2013-2018; el Programa Sectorial de Desarrollo Agrario, Territorial y Urbano, 2013-2018; el Programa Nacional de Vivienda 2014-2018; la NAMA de Vivienda; el Programa Nacional para el Aprovechamiento Sustentable de la Energía, 2014, entre otros.

${ }^{6}$ Sistema de Evaluación de Vivienda Verde.

${ }^{7}$ Acciones de Mitigación Nacionalmente Apropiadas, por sus siglas en inglés.

${ }^{8}$ El Sistema de Evaluación de la Vivienda Verde, Sisevive-Ecocasa, permite evaluar integralmente los elementos del diseño, las características constructivas y las tecnologías de cualquier vivienda ubicada en México. Asimismo, teniendo como 
eficiencia energética y el desempeño medioambiental de la vivienda a través de la incorporación de ecotecnias con estándares que garantizan la disminución de emisiones de GEI. Adicionalmente, en el mismo periodo se otorgaron alrededor de 1425000 créditos de Hipoteca Verde del Instituto del Fondo Nacional de la Vivienda para los Trabajadores $^{9}$ (SNIIV, 2018).

Para el caso de las ecotecnologías, las viviendas con calentadores solares de agua instalados en 2018 iban desde 2\% para la región cálida extrema, hasta $20 \%$ para la región templada (la Ciudad de México corresponde a esta última), y sólo $0.55 \%$ de los hogares de todo México en 2015 tenían paneles fotovoltaicos (INEGI, 2015; 2018).

Con todo lo anterior, y a pesar de que el Infonavit financia anualmente alrededor de 350000 viviendas con Hipoteca Verde en el país, ${ }^{10}$ la cobertura de los programas de carácter federal no tiene alcance significativo en la Ciudad de México (alrededor de 2\% para la Hipoteca Verde y menos de 1\% para el Sisevive), ${ }^{11}$ cuando $8.14 \%$ del total de viviendas del país se ubican en la entidad; es decir, en el año 2015 la ciudad tenía 2601323 viviendas (INEGI, 2015). Por su parte, el Instituto de Vivienda de la Ciudad de México había financiado alrededor de 40000 acciones para vivienda sustentable de 2012 a mediados de 2017, antes de enfocar sus acciones al otorgamiento de crédito para la reconstrucción de viviendas afectadas por el sismo del 19 de septiembre de 2017.

premisa el confort térmico y el consumo racional de agua, el sistema otorga una mejor calificación a las viviendas que tienen una menor demanda de energía y agua respecto a una vivienda de referencia. https://www.gob.mx/cms/uploads/attachment/ file/84276/SISEVIVECONUEE.pdf

${ }^{9}$ Resultados operativos de la Hipoteca Verde, consultado el 20 de marzo de 2019 en: http://portal.infonavit.org.mx/wps/wcm/connect/infonavit/cuentas+claras +transparencia/Informacion_obligatoria_art70/XXX+Estadisticas

${ }^{10}$ Información disponib̄e en Resultados operativos de la Hipoteca Verde, consultado el 20 de marzo de 2019 en http://portal.infonavit.org.mx/wps/wcm/connect/ infonavit/cuentas+claras+transparencia/Informacion_obligatoria_art70/XXX+Estadisticas

${ }^{11}$ Datos calculados por los autores, de la información disponible en Resultados operativos de la Hipoteca Verde, consultado el 20 de marzo de 2019 en: http:// portal.infonavit.org. $\mathrm{mx} / \mathrm{wps} / \mathrm{wcm} /$ connect/infonavit/cuentas+claras+transparencia/ Informacion_obligatoria_art70/XXX+Estadisticas, y http://sniiv.beta.conavi.gob.mx/ 
Las cifras que se muestran no son alentadoras cuando se sabe que de los 31.95 millones de viviendas que existían en México en 2015, 64.2\% fueron construidas por autoproducción; es decir, con el presupuesto, el control y la supervisión del propietario, sin financiamiento de los Organismos Nacionales de Vivienda o la banca comercial (Coneval, 2018). Si se considera que el mercado formal de viviendas tiene una baja cobertura (ibid.) y que aproximadamente el $43 \%$ de las que se venden son nuevas, se puede decir que sólo alrededor del 13\% del total de las viviendas que se producen en México tienen el potencial de poder implementar los programas que incluyen ecotecnologías, ${ }^{12}$ lo cual deja fuera a más del $80 \%$ de las viviendas que se producen en el país.

Lo que queda por hacer es pensar en ampliar la estrategia hacia modalidades como la adquisición de vivienda usada, el mejoramiento y la autoproducción, como se ha discutido en foros y mesas interinstitucionales sobre el tema. El principal obstáculo para que las viviendas nuevas por autoproducción y las viviendas usadas, así como las acciones de mejoramiento en las ya existentes, incorporen ecotecnologías, es que la decisión de instalar dichos dispositivos recae en la voluntad del usuario o propietario, sin que exista una forma de controlar, medir, regular o exigir por parte de las autoridades gubernamentales. De ahí la importancia de estudiar los factores que motivarían a que los usuarios conozcan, elijan, operen y mantengan las ecotecnologías para que el resultado de su adopción en el corto y mediano plazos no dependa de la industria inmobiliaria o de los programas gubernamentales.

Los esfuerzos en investigación y metodologías para la evaluación de la sustentabilidad en México (Morillón, 2009, 2011; Álvarez-Partida, 2013; Ceballos, 2013; NAMA, 2012; Morillón y Ceballos, 2017; Conavi, 2018) son muchos, pero insuficientes. Sin embargo, la mayoría de los trabajos se han dirigido a la evaluación y la certificación de las viviendas como unidades de análisis, y han

${ }^{12}$ Asimismo, no hay mecanismos de evaluación y seguimiento de Sisevive e Hipoteca Verde en los que se verifique que las viviendas equipadas con las ecotecnologías, en el mediano y largo plazos, mantengan los índices de desempeño global de eficiencia energética con los que fueron diseñados. 
quedado fuera del estudio los factores socioculturales involucrados en el desarrollo de la vivienda sustentable (Conavi, 2008), así como la percepción e interés de los usuarios para adoptar estas tecnologías ecológicas pues quienes finalmente habitan y mantienen la vivienda son los que pueden garantizar el cumplimiento de los objetivos a mediano y largo plazos (Uchida, 2005; Ortiz, Malagón y Masera, 2015). En este trabajo, las unidades de análisis no son las viviendas sino sus usuarios, como agentes que, de manera diferenciada por estrato socioeconómico, conocen, deciden, operan e incorporan o no las ecotecnologías a su vida cotidiana.

\section{La incorporación del usuario en el conocimiento y el uso de las ecotecnologías para la vivienda, desde la perspectiva del construccionismo social}

El construccionismo social (Berger y Luckmann, 2003) es una línea teórica que, desde la década de 1970, ha tratado de entender la relación ser humano-naturaleza como un constructo social, como un conjunto de prácticas cotidianas estructuradas a partir de conocimientos, símbolos, discursos y normas que surgen en la sociedad y que regulan las formas de actuar e interactuar de los agentes que pertenecen a ese grupo social, en un entorno ambiental específico (Lezama, 2002); por este motivo, las formas de reaccionar ante los problemas ambientales varían en función de la estructura de pensamiento y de los símbolos que cada agente interpreta de lo que la sociedad ha expresado como discurso.

Por lo tanto, un desafío ambiental se constituye como problema en el momento en el que un grupo social define la magnitud del daño que puede causar a esa misma sociedad, y se actúa en la medida en la que el sujeto interioriza el discurso o la demanda social. El mensaje se estructura internamente a través de símbolos, para que finalmente se decida actuar. La construcción de un problema ambiental se da en el ámbito de lo público, de la publicidad, de la difusión y de los medios; es ahí en donde se legitima. Por su parte, Kidd y Lee sugieren que 
[...] la preocupación y el apoyo a la protección ambiental es más bien producto de un paulatino cambio en los valores prioritarios, y no tanto el resultado de condiciones ambientales objetivas [Kidd y Lee, citados en Lezama, 2002, p. 403].

Sin embargo, el cambio en las prioridades de los agentes sucede por la interacción social; se trata de una actividad de construcción y reconstrucción constantes, de reflexión práctica.

Sería de esperarse que, en la medida en la que los sujetos tienen una percepción negativa del problema ambiental, o "conciencia ambiental", estarían en posibilidades de emprender acciones que mejoren la calidad del ambiente; sin embargo, el paso de la percepción de un problema a la acción que lo revierta no necesariamente ocurre de manera inmediata y concomitante. "La gente, en algunas ocasiones, elige ignorar los problemas porque les resulta sumamente difícil encontrar soluciones" (Lezama, 2002, p. 405). De acuerdo con Beck,

[...] la sensibilidad ecológica y la voluntad para protestar están influenciados por muchos factores: la densidad demográfica, el grado de industrialización, el nivel de prosperidad [...] pero están también influidos simplemente por normas culturales y formas de vida [Beck, 1995, p. 125].

Con todo esto, ¿cómo se transita exitosamente de un pensamiento o percepción a la acción? Las prácticas de los grupos sociales están basadas en sus propias formas de conocimiento, que a su vez estructuran las respuestas de los sujetos en relación con lo que es considerado como naturaleza. Desde el estructuralismo, el concepto de habitus

[...] asegura la presencia activa de las experiencias pasadas que, registradas en cada organismo bajo la forma de esquemas de percepción, de pensamientos y de acción, tienden, con más seguridad que todas las reglas formales y todas las normas explícitas, a garantizar la conformidad de las prácticas y su constancia a través del tiempo [Bordieu, 2007, p. 88]. 
Es el conjunto de esquemas de pensamiento aprendidos y construidos que se expresan de manera práctica en el mundo, con los límites que le han sido asignados socialmente.

Las formas de respuesta a las problemáticas ambientales serán diversas como expresión de la construcción social de las diferentes interacciones que ocurren entre grupos; esta interacción expresa simultáneamente "el orden jerárquico de las necesidades del grupo de acuerdo con sus condiciones socioeconómicas" (Lezama, 2002, p. 403). Si están satisfechas las necesidades básicas, entonces surgirán otras. Es un proceso constante de readecuación de la escala de valores, en el que están involucradas las generaciones y la edad de los agentes.

En otro orden de ideas, es importante señalar que la población que habita en las ciudades no constituye un todo homogéneo, cuyos intereses y objetivos están en sincronía bajo supuestos ideales de comunidad y búsqueda del bien común, ya que los grupos sociales se constituyen de acuerdo con variantes sociales, culturales, económicas y territoriales que moldean y definen sus intereses, objetivos o metas de manera diferenciada. Entonces, podría decirse que los diversos grupos sociales urbanos construyen percepciones, discursos y normas diferentes sobre un problema ambiental, y con base en las formas interiorizadas y convenidas socialmente por cada grupo, se desarrollará la forma en la que reaccionen ante dicho problema ambiental. Las diferencias en las formas de habitar dependerán también "de la posición social que cada individuo logra ocupar en la estructura de la ciudad" (Cortés, 1995, p. 9).

Las bases teóricas que moldean este trabajo, y que buscan ser comprobadas empíricamente, se refieren a que diferentes grupos sociales de la Ciudad de México construyen socialmente, a través de narrativas y normas culturales, una percepción sobre la problemática ambiental de la urbe, que motiva a que socialmente se construyan formas distintas de conocimiento en torno a estrategias y prácticas que minimicen el impacto de las actividades en las viviendas sobre el ambiente. 


\section{Metodología}

La hipótesis que guio el desarrollo de este trabajo consiste en la idea de que el conocimiento y el uso de las ecotecnologías para la vivienda en la Ciudad de México están determinados por las formas de percepción de la calidad del ambiente en la urbe, la edad, la escolaridad y los estratos socioeconómicos de los diferentes grupos sociales que habitan la metrópoli.

Con base en estos supuestos, se diseñó un estudio transversal cuantitativo y correlacional en el que se definen como variables independientes: 1) el estrato socioeconómico y territorial, 2) el nivel de escolaridad, 3) la edad del entrevistado, y 4) la percepción de la calidad ambiental de la Ciudad de México. Las variables dependientes son: a) el conocimiento de las ecotecnologías, y b) su uso.

La recolección de datos se hizo a través de una muestra aleatoria estratificada proporcional ${ }^{13}$ en cinco estratos, categorizados por un análisis que combinó las variables de uso y precio de suelo, densidad poblacional e índice de marginación. Con esto, se obtuvieron los siguientes cinco estratos socioeconómicos y territoriales en la Ciudad de México: urbano alto, urbano medio, urbano bajo, periurbano y rural. ${ }^{14}$ Se identificaron diez sitios, ${ }^{15}$ dos para cada estrato.

${ }^{13}$ La ecuación del modelo fue $\mathrm{n}=\mathrm{Z1} 1-\alpha 2 * \mathrm{p} * 1-\mathrm{p} 2=\mathrm{n}=1.642(0.5)(1-0.5)$ $(0.15) 2=29.88 \approx 30$; por sitio de muestreo, se eligió una muestra de entrevistados de tipo aleatoria simple, con un error de muestreo $\varepsilon=15.0 \%$; nivel de confianza de $90 \%(Z 1-\alpha=1.64)$ y prevalencia de $(\mathrm{p}=0.5)$ (Schelesselman, 1982).

${ }^{14}$ Este tipo de clasificación no se reduce simplemente a las condiciones socioeconómicas de la población; también incluye configuraciones espaciales diferenciadas que expresan estructuras culturales particulares de los grupos sociales, como los casos de los rurales y los periurbanos.

${ }^{15}$ Los sitios de muestreo fueron lugares públicos dentro de las colonias definidas por estratos, excepto en el caso de los rurales, en los que la encuesta se aplicó directamente en los hogares. Los sitios en donde se aplicaron las encuestas fueron: Santa Fe, alcaldía Cuajimalpa; Polanco, alcaldía Miguel Hidalgo; Centro, alcaldía Coyoacán; Condesa, alcaldía Cuauhtémoc; San Lorenzo Tezonco, alcaldía Iztapalapa; Colonia Proletaria, alcaldía Gustavo A. Madero; San Gregorio Atlapulco, alcaldía Xochimilco; San Nicolás Tetelco, alcaldía Tláhuac; Paraje Oluca, alcaldía Milpa Alta; y El Encinal, alcaldía Milpa Alta. 


\section{Mapa 1}

Estratificación socioespacial de la Ciudad de México*

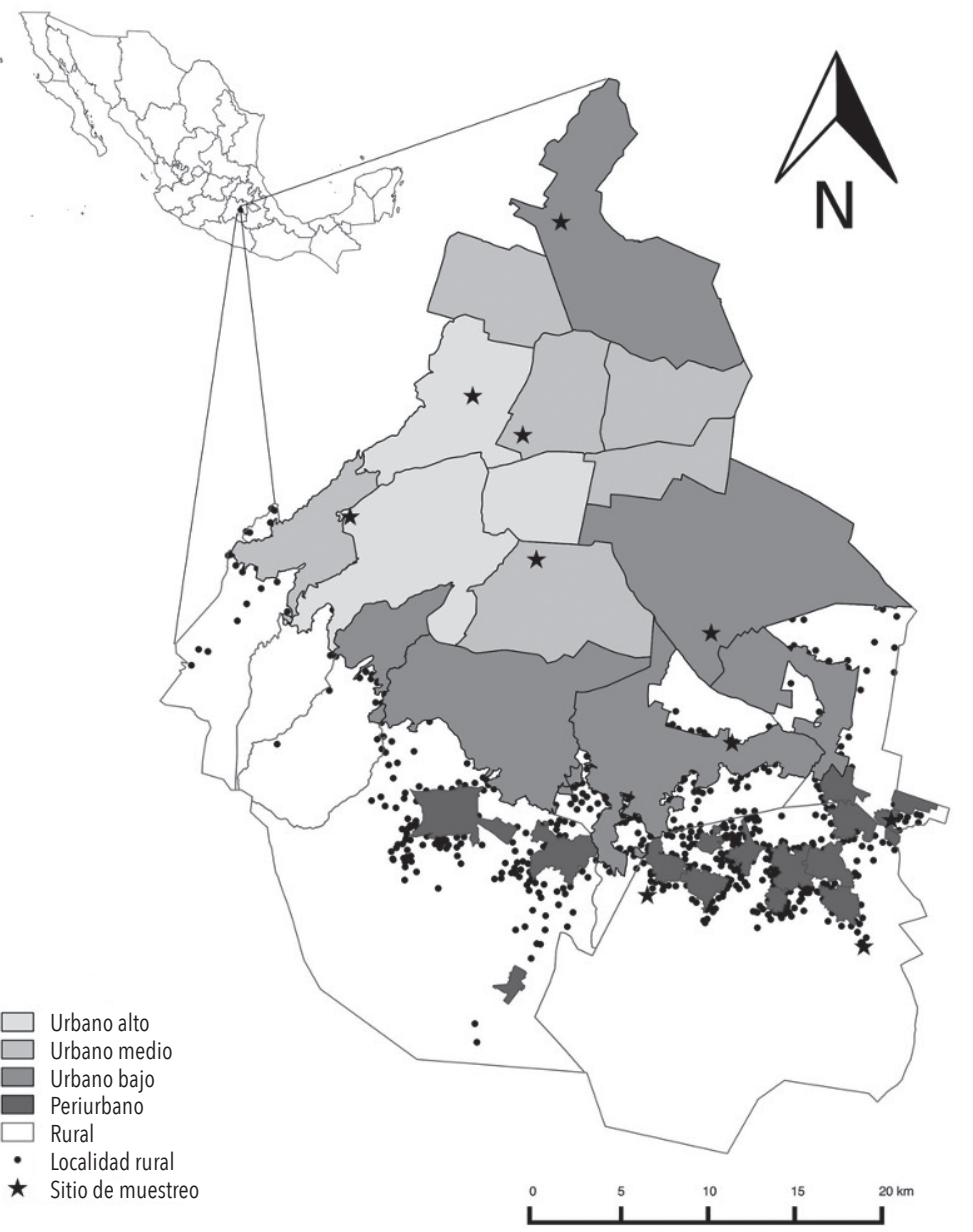

* La unidad de análisis territorial para los estratos corresponde con las alcaldías de la Ciudad de México.

Fuente: Elaboración propia con datos de INEGI (2010) y ALDF (2014). 
Los cuestionarios aplicados incluyeron 22 preguntas sobre características generales de los encuestados (edad, escolaridad y ocupación), características generales de sus viviendas, percepción de la calidad del ambiente en la ciudad, conocimiento de ecotecnologías y uso de dispositivos con grado ecológico. Se aplicaron 300 cuestionarios en los meses de octubre y noviembre de 2015.

Las variables independientes se determinaron de la siguiente manera: el estrato socioeconómico quedó definido desde el diseño de la muestra; para el caso del análisis estadístico, en el que se requerían variables cuantitativas, se utilizó el precio de suelo como índice de estrato socioeconómico; la edad y la escolaridad se trabajaron en rangos quinquenales de años y como niveles educativos completados; respecto a la evaluación de la calidad del medio ambiente en la urbe, se preguntó directamente a los encuestados qué calificación le daban a la calidad del ambiente de la ciudad en un rango de uno a diez.

En cuanto a las variables dependientes, el conocimiento de las ecotecnologías fue evaluado a partir del reconocimiento de la variedad de sistemas y dispositivos que los encuestados identificaban o reconocían dentro de un listado de catorce ecotecnologías. No se evaluó el nivel de conocimiento técnico sobre el funcionamiento de cada una de las técnicas referidas, sino el número de ecotecnologías que el entrevistado refirió identificar en una escala de cero a diez; en referencia a la evaluación del uso de ecotecnologías, se preguntó directamente cuántos de los dispositivos y sistemas antes mencionados tenían instalados en sus viviendas.

Para probar la hipótesis propuesta de que el estrato socioeconómico y territorial, el nivel de escolaridad, la edad y la percepción de la calidad ambiental de la ciudad determinan el conocimiento y el uso de las ecotecnologías, primero se realizaron dos análisis para determinar el tipo de relación entre las variables independientes y las dependientes por medio de dos pruebas estadísticas: un análisis de asociación Ji-cuadrada (Cortés y Rubalcava, 1987) y uno de correlación. Posteriormente, se hizo un análisis de regresión lineal múltiple con cuatro modelos que permitirán identificar la intensidad de las variables independientes y aportar a la varianza total el conocimiento de los encuestados sobre las ecotecnologías para la vivienda. 


\section{Resultados y discusión}

En general, el promedio de conocimiento en la muestra analizada sobre ecotecnologías para la vivienda es de 6.32 puntos en una escala de cero a diez. Se observó que cada encuestado tiene instalada menos de dos ecotecnologías (1.72) en sus viviendas, y en ocho de cada diez casos utilizan focos ahorradores en sus casas. Los hallazgos sobre las variables dependientes se presentan a continuación.

\subsection{Conocimiento de las ecotecnologías para la vivienda en la Ciudad de México}

El resultado muestra que el conocimiento de las ecotecnologías para la vivienda se vincula significativamente con el estrato socioeconómico y territorial, así como con el nivel de escolaridad de los encuestados, tanto en las pruebas de Ji-cuadrada como en la de correlación. En el caso de la edad y la percepción de la calidad ambiental de la ciudad, no existe relación significativa sobre el conocimiento de las ecotecnologías para la vivienda en los sitios de muestreo.

La prueba de regresión lineal múltiple reveló que en uno de los modelos con las variables precio de suelo, años de escuela y edad, se explica el $29.9 \%$ de la varianza sobre el conocimiento. Sin embargo, el conocimiento de las ecotecnologías para la vivienda se expresa sólo a través de las variables precio de suelo y años de escolaridad ( $\mathrm{f}=63.47$ y $\mathrm{p}=0.001$ ); con estos resultados se observó que la edad y la percepción de la calidad de medio ambiente en la ciudad no aportan significativamente al conocimiento de las ecotecnologías para la vivienda.

Con esta prueba se evidenció que, a mayor estrato socioeconómico, es más probable que los entrevistados tengan niveles de escolaridad más altos, por lo que se esperaría que la población que reside en los estratos medios y altos con mayor nivel educativo, tenga más conocimiento sobre las ecotecnologías para la vivienda, a diferencia de quienes pertenecen a los estratos socioeconómicos bajo, rural o periurbano y que cuentan con pocos años destinados al estudio. 


\section{Cuadro 1}

Variables vinculadas estadísticamente con el conocimiento de ecotecnologías para la vivienda

\begin{tabular}{|c|c|c|c|c|}
\hline \multirow[b]{2}{*}{ Variable } & \multicolumn{2}{|c|}{ Análisis de asociación ${ }^{a}$} & \multicolumn{2}{|c|}{ Análisis de correlación ${ }^{b}$} \\
\hline & $\begin{array}{c}\text { Ji-cuadrada } \\
\text { Pearson }\end{array}$ & $\begin{array}{c}\text { Valor } * \\
P\end{array}$ & $\begin{array}{c}\text { Correlación } \\
\text { Pearson }\end{array}$ & $\begin{array}{c}\text { Valor }^{* * * *} \\
\boldsymbol{P}\end{array}$ \\
\hline $\begin{array}{l}\text { Estrato socioeconómico } \\
\text { y territorial }\end{array}$ & 107.78 & $0.000 * *$ & 0.412 & $0.000 * *$ \\
\hline Escolaridad & 184.06 & $0.000 * *$ & 0.542 & $0.000 * *$ \\
\hline Edad & 147.41 & 0.141 & -0.002 & 0.969 \\
\hline $\begin{array}{l}\text { Percepción calidad } \\
\text { ambiental ciudad }\end{array}$ & 166.25 & 0.272 & -0.079 & 0.171 \\
\hline
\end{tabular}

* Asintótica bilateral.

** Valor significativo cuando $\mathrm{P} \leq 0.05$.

*** Valor de significación en el nivel 0.01 bilateral.

${ }^{\text {a }}$ Con variables cualitativas ordinales

${ }^{\mathrm{b}}$ Para variables cuantitativas (precio del suelo, años de escolaridad, edad y percepción de la calidad ambiental de la ciudad).

Nota: $n=300$.

Fuente: Elaboración propia con datos de campo.

Para identificar las fuentes o los medios de información a través de los cuales se adquiere el conocimiento de las ecotecnologías, en el Cuadro 2 se presentan los resultados en los que se puede apreciar que los encuestados que más ecotecnologías conocen (37.3\%), lo lograron a través de los medios de difusión, internet y redes sociales; en segundo lugar, por su contacto directo en los centros de comercialización o porque fueron instalados en la vivienda de algún familiar o conocido (27.0\%); además, el 17\% de los encuestados comparten y socializan su experiencia con el uso de las ecotecnologías, lo que contribuye directamente con la construcción social del uso de éstas (Berger y Luckmann, 2003). Otro resultado importante es que la escuela sólo representa el $7.7 \%$ de las formas a través de las cuales los encuestados adquirieron este conocimiento, por lo que ésta no constituye un medio de difusión de conocimiento sobre el tema, sino que los años de escolaridad otorgan a los sujetos la posibilidad de tener acceso a formas diferentes de conocer e investigar sobre ecotecnologías, más allá del conocimiento que pueden llegar a obtener a través de la escuela. 


\section{Cuadro 2}

Nivel de conocimiento de ecotecnologías para la vivienda, por fuente de aprendizaje (porcentajes)*

\begin{tabular}{lrrrrr}
\hline & \multicolumn{5}{c}{ Conocimiento de ecotecnologías } \\
\cline { 2 - 6 } Fuente de aprendizaje & Nulo & Bajo & Medio & Alto & Total \\
\hline Medios de comunicación, & 1.00 & 4.70 & 19.70 & 12.00 & 37.30 \\
internet y redes sociales & & & & & \\
Físicamente & 3.30 & 9.00 & 10.70 & 4.00 & 27.00 \\
Talleres y exposiciones & 0.00 & 0.30 & 1.30 & 0.00 & 1.70 \\
Interacción social & 0.30 & 2.30 & 9.00 & 5.30 & 17.00 \\
Escuela & 0.00 & 1.30 & 3.70 & 2.70 & 7.70 \\
Trabajo & 0.00 & 0.30 & 2.00 & 6.30 & 8.70 \\
Programas de gobierno* & 0.00 & 0.00 & 0.00 & 0.30 & 0.30 \\
Total & 4.70 & 18.00 & 46.30 & 31.00 & 100.00 \\
\hline
\end{tabular}

* Incluyen los programas de Hipoteca Verde y del INVI.

Nota: $n=300$.

Fuente: Elaboración propia con datos de campo.

\subsection{Uso de ecotecnologías para la vivienda}

En lo que respecta a la práctica y utilización cotidiana de las ecotecnologías, los resultados de asociación y correlación de variables son similares a los obtenidos en cuanto al conocimiento, ya que se encontró que el uso de ecotecnologías está significativamente relacionado con el estrato socioeconómico y territorial, así como con el nivel de escolaridad de los encuestados, a diferencia de las variables edad o percepción sobre la calidad del medio ambiente, cuyos resultados muestran que no existe asociación de ellas con el uso de las ecotecnologías en las viviendas.

Al realizar el análisis de regresión lineal múltiple con cuatro modelos, se observó que sólo el $15 \%$ de la varianza del uso de las ecotecnologías en la vivienda se explica por las variables de precio del suelo y años de escolaridad de los usuarios, mientras que la edad y la percepción de la calidad ambiental de la ciudad no aportan al modelo. Con esto se buscó integrar un nuevo análisis de correlación para 
determinar si el uso de las ecotecnologías está determinado significativamente por el conocimiento de ellas. El resultado $\left(\mathrm{r}_{\mathrm{xy}}=0.366\right.$, $\mathrm{p}=0.000)$ muestra que sí existe una relación, aunque débil, entre el conocimiento y el uso.

\section{Cuadro 3}

Variables vinculadas estadísticamente

con el uso de ecotecnologías para la vivienda

\begin{tabular}{|c|c|c|c|c|}
\hline \multirow[b]{2}{*}{ Variable } & \multicolumn{2}{|c|}{ Análisis de asociación ${ }^{a}$} & \multicolumn{2}{|c|}{ Análisis de correlación ${ }^{b}$} \\
\hline & $\begin{array}{l}\text { Ji-cuadrada } \\
\text { de Pearson }\end{array}$ & Valor $P$ & $\begin{array}{l}\text { Correlación } \\
\text { de Pearson }\end{array}$ & Valor $P$ \\
\hline $\begin{array}{l}\text { Estrato socioeconómico } \\
\text { y territorial }\end{array}$ & 81.86 & $0.000 * *$ & 0.359 & $0.000^{* *}$ \\
\hline Escolaridad & 81.94 & $0.000 * *$ & 0.359 & $0.000^{* *}$ \\
\hline Edad & 69.26 & 0.503 & -0.005 & 0.929 \\
\hline $\begin{array}{l}\text { Percepción calidad } \\
\text { ambiental ciudad }\end{array}$ & 91.68 & 0.265 & -0.053 & 0.361 \\
\hline
\end{tabular}

* Asintótica bilateral.

** Valor significativo cuando $\mathrm{P} \leq 0.05$.

$* * *$ Valor de significación en el nivel 0.01 bilateral.

${ }^{a}$ Con variables cualitativas ordinales.

b Para variables cuantitativas (precio del suelo, años de escolaridad, edad y percepción de la calidad ambiental de la ciudad).

Fuente: Elaboración propia con datos de campo.

En un segundo análisis de regresión lineal múltiple se observó que el conocimiento de las ecotecnologías, precio del suelo y años de escolaridad explican el $18.4 \%$ de la variabilidad en su uso.

En el Cuadro 4 se muestran los porcentajes obtenidos de la variable número de ecotecnologías utilizadas por estrato socioeconómico. Se puede observar que los encuestados que habitan en la porción rural de la Ciudad de México son quienes en mayor proporción carecen de alguna ecotecnología en su vivienda, seguidos por el estrato urbano bajo. En la porción periurbana de la urbe se encontró mayor porcentaje de población con ecotecnologías en la vivienda, en comparación con los estratos anteriormente mencionados. Se confirman los resultados de la correlación estadística en la que los estratos con un poder adquisitivo y precios del suelo alto son quienes tienden 
a utilizar un mayor número de ecotecnologías en sus viviendas, en comparación con los estratos rural y urbano bajo.

\section{Cuadro 4}

Número de ecotecnologías instaladas en las viviendas por estrato socioeconómico y territorial (porcentajes)

\begin{tabular}{|c|c|c|c|c|c|c|}
\hline $\begin{array}{l}\text { Núm. eco- } \\
\text { tecnologías } \\
\text { utilizadas }\end{array}$ & $\begin{array}{c}\text { Urbano } \\
\text { alto }\end{array}$ & $\begin{array}{c}\text { Urbano } \\
\text { medio }\end{array}$ & $\begin{array}{c}\text { Urbano } \\
\text { bаjo }\end{array}$ & Periurbano & Rural & Total \\
\hline No tiene & 4.7 & 5.7 & 12.7 & 11.7 & 16.3 & 51.0 \\
\hline 1 a 3 & 13.3 & 14.0 & 7.3 & 7.7 & 3.7 & 46.0 \\
\hline 4 a 6 & 1.7 & 0.3 & 0.0 & 0.7 & 0.0 & 2.7 \\
\hline Más de 7 & 0.3 & 0.0 & 0.0 & 0.0 & 0.0 & 0.3 \\
\hline Total & 20.0 & 20.0 & 20.0 & 20.0 & 20.0 & 100.0 \\
\hline
\end{tabular}

Nota: $n=300$.

Fuente: Elaboración propia con datos de campo.

\section{Cuadro 5}

Número de ecotecnologías instaladas en las viviendas por nivel educativo de los usuarios (porcentajes)

\begin{tabular}{lccccc}
\hline \multirow{2}{*}{$\begin{array}{l}\text { Nivel } \\
\text { educativo }\end{array}$} & No tiene & $\mathbf{1} \boldsymbol{a} \mathbf{3}$ & $\mathbf{4} \boldsymbol{a} \mathbf{~}$ & Más de & Total \\
\cline { 2 - 6 } Primaria & 13.7 & 2.3 & 0.0 & 0.0 & 16.0 \\
Secundaria & 12.7 & 6.0 & 0.3 & 0.0 & 19.0 \\
Preparatoria & 11.7 & 11.0 & 0.7 & 0.0 & 23.3 \\
Universidad & 9.0 & 19.3 & 1.0 & 0.0 & 29.3 \\
Posgrado & 2.0 & 7.0 & 0.7 & 0.3 & 10.0 \\
No estudió & 2.0 & 0.3 & 0.0 & 0.0 & 2.3 \\
Total & 51.0 & 46.0 & 2.7 & 0.3 & 100.0 \\
\hline
\end{tabular}

Nota: $n=300$.

Fuente: Elaboración propia con datos de campo.

Respecto al nivel educativo (Cuadro 5), que fue otra de las variables significativas en la utilización de ecotecnologías, se observa que más de la mitad de los encuestados no usa ninguna en su vivienda aparte de los focos ahorradores, y $46.0 \%$ de las personas 
tienen entre una y tres ecotecnologías en sus moradas. El mayor porcentaje lo ocupan las personas con nivel de estudios universitarios. Sin embargo, se observa que las personas que no estudiaron y las que sólo pudieron completar la primaria, llegan a tener como máximo tres ecotecnologías, a diferencia de los otros estratos, con casos en los que tienen hasta seis o siete entre quienes tienen nivel de posgrado.

\subsection{Uso de ecotecnologías por estrato}

En el Cuadro 6 es posible desagregar porcentajes de uso por ecotecnología y por estrato, los cuales se explican a continuación.

a) Urbano alto. Las principales ecotecnologías que este estrato utiliza, en orden de frecuencia, son: sistemas ahorradores de agua, calentadores de paso, purificadores de agua y calentador solar. Éste es el estrato con menor uso de focos ahorradores (siete de cada diez casos). Tiene el mayor número de uso de celdas y calentadores solares, así como mayor proporción de sistemas ahorradores de agua. Es el único estrato que utiliza materiales de construcción ecológicos y diseño bioclimático en las viviendas. La principal motivación por la que los encuestados de este estrato instalan ecotecnologías en sus viviendas es el ahorro en los servicios (37\%), seguido de la preocupación por el ambiente (22\%).

b) Urbano medio. Este estrato tiene un comportamiento similar a los resultados de toda la muestra, ya que, en la mayoría de los casos, se obtienen valores cercanos a la media del estudio. Las ecotecnologías más utilizadas por los usuarios de este estrato son: calentadores de paso, sistemas ahorradores de agua, purificadores de agua y composta. Utilizan también las celdas solares, aunque en baja proporción. Este estrato es el que mayor proporción tiene en la implementación de huertos urbanos. Tiene su mayor motivación para el uso de ecotecnologías en sus viviendas el ahorro en servicios (43\%), seguido por la preocupación por el medio ambiente (17\%). En uno de cada diez encuestados de este estrato, los dispositivos ya estaban incluidos en las viviendas en las que habitan. Resalta el hecho de que, en el urbano medio, la principal justificación de no usar ecotec- 
nologías es la falta de motivación porque el costo de los servicios es bajo y no se percibe la necesidad de cambiar.

c) Urbano bajo. En este estrato se encontraron las más altas proporciones de utilización de focos ahorradores en toda la muestra. Las ecotecnologías que más frecuentemente se usan son los calentadores de paso y los sistemas ahorradores de agua. Quienes pertenecen a este nivel, no han incorporado a sus viviendas ningún sistema de climatización natural ni de manejo de residuos. En general, no utilizan sistemas de funcionamiento pasivo. Como puede observarse, este estrato está motivado a utilizar ecotecnologías para ahorrar en el consumo de agua, gas y energía eléctrica (62\%); la preocupación por el ambiente cae al quinto lugar, con tan sólo 3.3\%. En este estrato utilizan las ecotecnologías porque consideran que son las disponibles en el mercado ya que no hay opción (sobre todo cuando se refieren a los focos ahorradores). La principal causa por la que $63 \%$ no tiene ecotecnologías diferentes a los focos ahorradores en sus casas es porque rentan la vivienda en la que habitan.

d) Periurbano. Este estrato utiliza con mayor frecuencia los sistemas ahorradores de agua, el calentador solar, el calentador de paso y los sistemas de recolección de agua de lluvia. Se observa un valor superior a la media general en el uso de celdas solares. El valor resultante en el uso de los calentadores solares es superior a la media general y cercano al valor de los estratos urbanos altos. Resalta también que es el único estrato con uso de estufa solar y el más alto en el empleo de sistemas captadores de agua de lluvia, baños secos y composta, caracterizados por tener un tipo de funcionamiento pasivo. El motivo por el que usan las ecotecnologías es el ahorro en servicios (53\%), seguido por el que ya no hay otra alternativa en el mercado $(17 \%) \mathrm{y}$, por último, la preocupación por el ambiente $(8.3 \%)$.

e) Rural. Éste es el estrato con menor proporción de uso de ecotecnologías; sin embargo, los sistemas más utilizados, aunque sea en proporciones bajas (siete y ocho entre cien), son: el boiler de paso, los sistemas de captación de agua de lluvia y los ahorradores de agua potable. Por lo general, sólo utilizan focos ahorradores debido a que son más eficientes para resistir los cambios de voltaje ocasionados por la variabilidad de la corriente que tienen, debido a que nadie en 
las localidades tiene servicio de electricidad de manera formal. Los resultados señalan que $18.3 \%$ aún utilizan leña como combustible, que obtienen de los bosques cercanos del suelo de conservación de la Ciudad de México. También se observó que este estrato no utiliza sistemas con funcionamiento pasivo, con excepción de los baños secos (en menos de dos casos por cada cien). El hecho de que no haya otra opción para el uso de focos ahorradores (25\%), junto con la motivación para ahorrar $(35 \%)$, son las principales razones por las que este estrato incorpora ecotecnologías a sus viviendas. Sólo 3.3\% de los encuestados refirieron que la calidad del medio ambiente es un motivo para tener ecotecnologías. El $81.7 \%$ no utiliza dispositivos de grado ecológico porque no tienen recursos económicos para comprarlos, además de que no cuentan con la seguridad de la propiedad en la que habitan.

\section{Conclusiones}

Para responder a la pregunta inicial, ¿cómo cambia el conocimiento y el uso de ecotecnologías en las viviendas existentes en la Ciudad de México, a partir de las diferencias socioterritoriales de los usuarios?, se puede decir que los sujetos que pertenecen a los estratos socioeconómicos urbano alto y medio son quienes en general más conocen y usan las ecotecnologías para las viviendas; ello está asociado a los niveles altos de escolaridad (licenciatura o posgrado).

Al contrastar los resultados obtenidos empíricamente con la teoría de la construcción social, se puede afirmar que sí existe un discurso y conocimiento diferenciado de las ecotecnologías para la vivienda por estrato socioeconómico y nivel escolar, no así por grupos de edad. Es decir, los estratos socioeconómicos urbano alto y urbano medio construyen socialmente discursos y normas culturales que estructuran los pensamientos y las percepciones de los sujetos, lo que les facilita el acceso a una mayor cantidad de recursos no sólo económicos, sino también a información sobre las ecotecnologías para la vivienda, lo que los motiva a implementarlas en mayor proporción en comparación con los otros grupos analizados. 


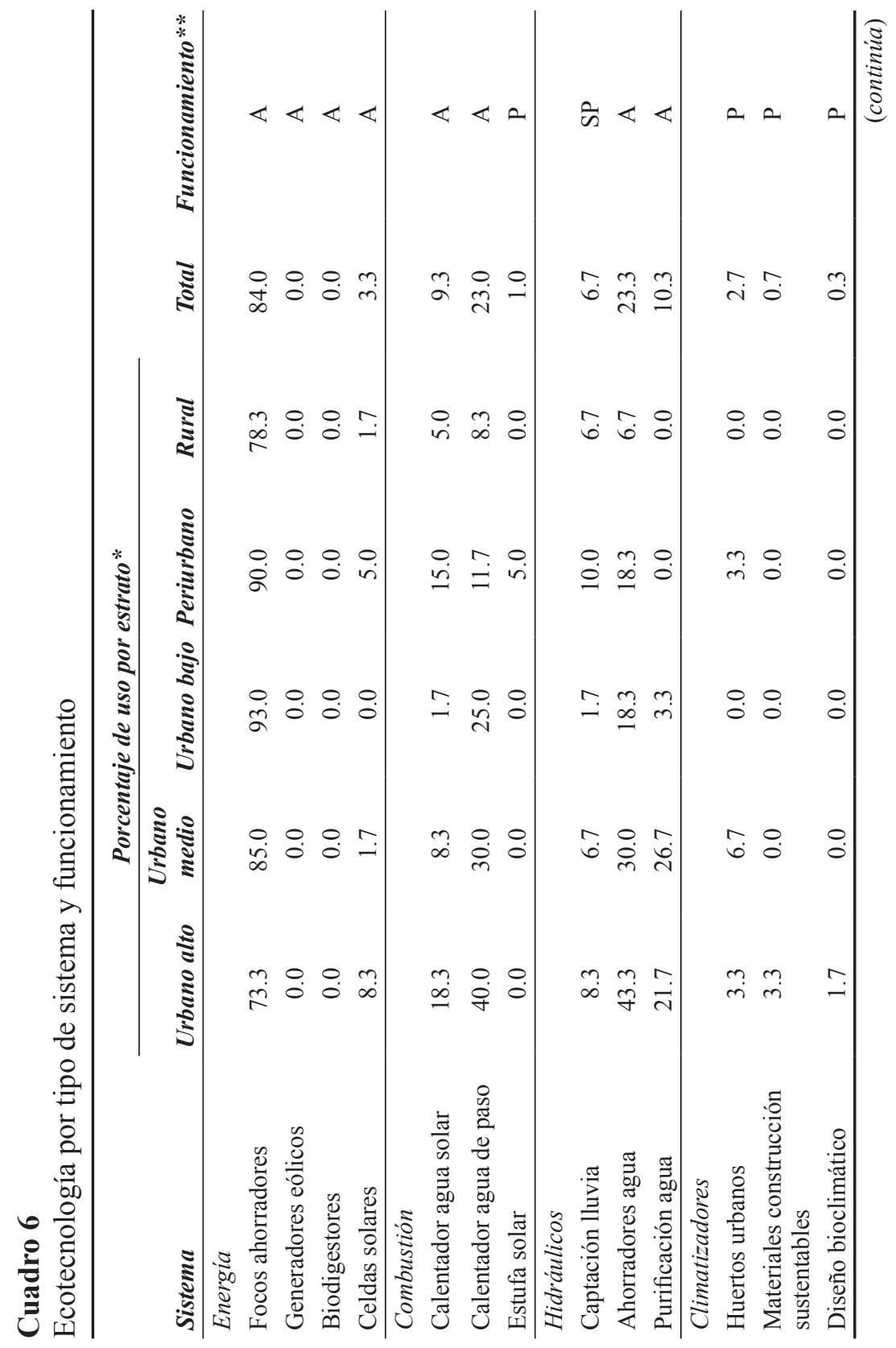




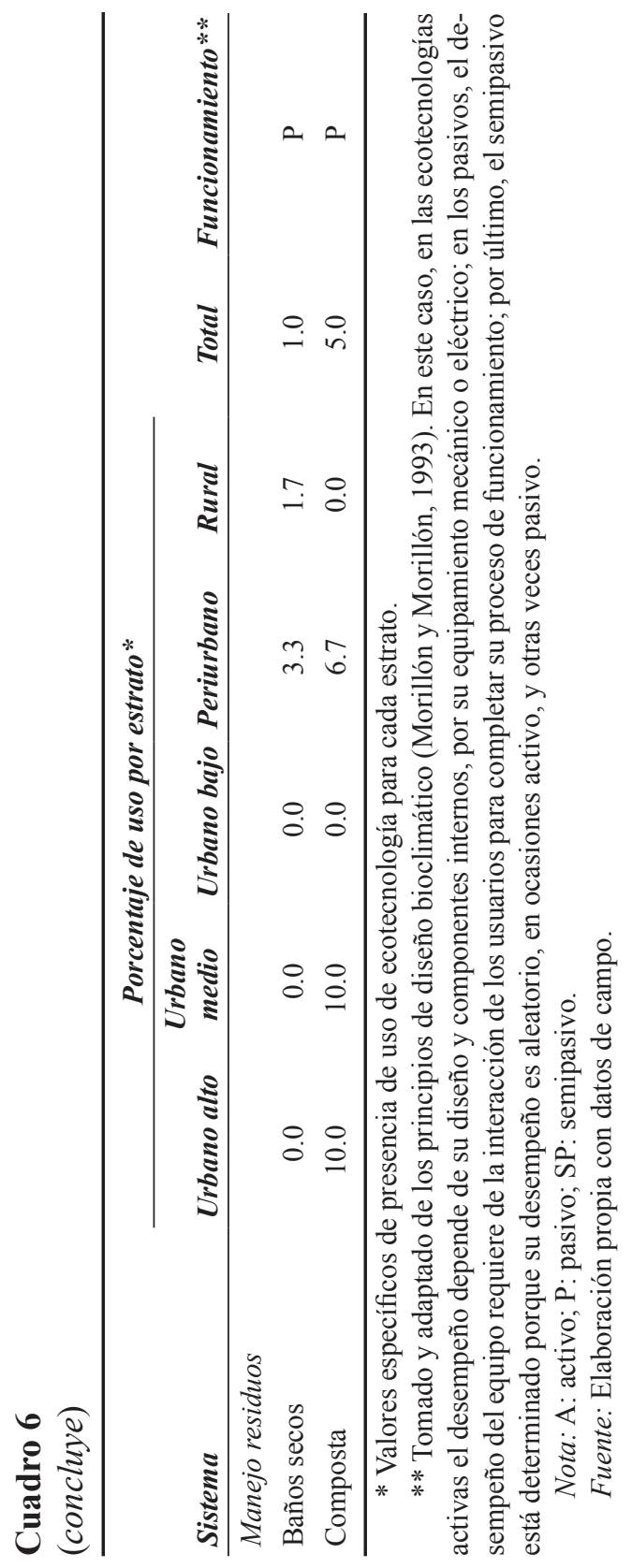


Para el estrato urbano alto, la escala de valores va más allá de la resolución de la manutención diaria, por lo que sus integrantes tienen la posibilidad de construir e incorporar lentamente -a través de discursos y normas sociales- valores sobre el cuidado del ambiente; cuentan con recursos económicos, entre otros, que les permiten arriesgarse a probar algo nuevo.

En el estrato urbano medio se encontró que existe una importante polarización. Por un lado, algunos sectores de este grupo consideran prioritario el cuidado ambiental y el uso de ecotecnologías para la vivienda en la medida en la que sus recursos económicos se los permiten, por lo que la mayoría de ellos utiliza sistemas activos disponibles en el mercado y accesibles en costo, así como sistemas pasivos poco complejos (como huertos urbanos). Sin embargo, como se observó, una porción de los integrantes del estrato urbano medio reproduce formas de vida condicionada que les permiten estar conformes con la manera económica en la que resuelven el acceso a servicios como la luz y el agua; por lo tanto, en su escala de valores aún no se registra la incorporación del cuidado del ambiente a través del uso de las ecotecnologías.

En cuanto a los estratos socioeconómicos urbano bajo y rural, están integrados por grupos sociales que han experimentado procesos históricos de acumulación de desventajas y de exclusión, lo que de igual forma se expresa en su forma limitada de conocimiento y acceso a las ecotecnologías para la vivienda. Su situación socioeconómica los obliga a resolver, a través de los medios de los que disponen, la satisfacción de sus necesidades básicas, aunado al desgaste físico resultado del tipo de trabajo que desempeñan y a que generalmente requieren transportarse a lejanos lugares de empleo. No cuentan con los recursos materiales que les permitan arriesgar o invertir en nuevos sistemas amigables con el ambiente, por lo tanto, únicamente recurren al uso de ecotecnologías caracterizadas por un funcionamiento activo y de bajo costo en el mercado. Quienes pertenecen al estrato urbano bajo sólo utilizan dispositivos tecnificados que les ayudan a ahorrar servicios disponibles regularmente, como agua, luz y gas. Por su parte, los del estrato rural no tienen servicios, por lo que las ecotecnologías les permiten eficientar los servicios de los que pueden allegarse de forma ilegal y precaria. 
Por otra parte, en el estrato periurbano se está generando un proceso lento de construcción social, en el que las ecotecnologías empiezan a tener mayor impacto, pues se están convirtiendo en un elemento cotidiano de lo que se dice y lo que se usa. Han encontrado además que las ecotecnologías les permiten solucionar problemas de escasez de servicios, de manera similar a como se han estado implementando en el medio rural en México. Quienes integran este estrato han encontrado que, por medio de la autoconstrucción y experimentación, pueden solucionar los problemas de falta de drenaje y servicio de agua potable; por ello, constituyen el estrato con mayor uso de sistemas con funcionamiento pasivo, como baños secos, captación de agua de lluvia y estufa solar. A diferencia del estrato rural, los encuestados periurbanos tienen mayor seguridad sobre la propiedad de la tierra, ya que no ocupan terrenos dentro de suelo con uso de conservación ambiental.

Por último, se sugiere que en estudios similares se procure incluir las diferencias por género. Adicionalmente, se recomienda introducir variables que expresen las preferencias del uso de ecotecnologías en las viviendas de los sujetos por grupo social, por ejemplo, la propiedad de la vivienda, las prioridades en la distribución cotidiana del ingreso, las percepciones sobre dispositivos tecnológicos fundamentales en la vivienda, el tiempo de uso de cada ecotecnología, entre otros; de tal manera que se puedan identificar la multiplicidad de elementos que inciden en la transformación en las escalas de valoración individual, construidas por los grupos sociales a los que pertenecen. Por otro lado, si se quiere promover un cambio para incrementar la adopción de nuevos sistemas tecnológicos amigables con el ambiente, no es suficiente con desarrollar formas de incentivar financieramente a los usuarios, sino también hay que considerar la desigualdad en el acceso a los recursos por parte de los diferentes grupos sociales, con el objetivo de incentivar la inclusión y el acceso de ecotecnologías para la vivienda en todos ellos.

Simultáneamente, en todos los estratos es importante trabajar discursivamente como sociedad en la construcción de formas de percepción, pensamiento y acción para incidir en la escala de valores, y trabajar conjuntamente en la difusión de un imaginario de vivienda con ecotecnologías en regiones bioclimáticas y contextos específi- 
cos. Es importante, además, que la divulgación sea el resultado de compartir las experiencias y recomendaciones de los propios usuarios. El cambio debe provenir de una estructura de pensamiento integrado por parte de los sujetos, para que por sí mismos busquen implementar sistemas sustentables a sus viviendas y estén dispuestos a asumir los costos (monetarios y culturales) implicados en los procesos de instalación, mantenimiento y reposición. Se considera que el principal reto estriba en que todos los actores involucrados trabajen conjuntamente en construir socialmente formas que produzcan paulatinamente cambios en la forma en la que la sociedad concibe, conoce y actúa respecto a las ecotecnologías para la vivienda.

\section{Bibliografía}

Álvarez-Partida, F. (2013). Criterios e indicadores sociales y económicos para la vivienda sustentable en México. Memorias del Congreso Nacional de Vivienda. Eje Temático 5. Medio Ambiente y Vulnerabilidad. Ciudad de México: UNAM.

Asamblea Legislativa del Distrito Federal (ALDF). (2014). Código Financiero 2014. Ciudad de México: Gobierno del Distrito Federal.

Beck, U. (1995). Ecological politics in an age of risk. Cambridge: Polity Press.

Berger, P. y Luckmann, T. (2003). La construcción social de la realidad. Buenos Aires: Amorrortu.

Bourdieu, P. (2007). El sentido práctico. Buenos Aires: Siglo XXI.

Carabias, J. y Landa, R. (2005). Agua, medio ambiente y sociedad: hacia la gestión integral de los recursos hídricos en México. Ciudad de México: Universidad Nacional Autónoma de México / El Colegio de México, A.C. / Fundación Gonzalo Río Arronte. Comisión para la Cooperación Ambiental (CCA). (2008). Edificación sustentable para América del Norte. Oportunidades y retos. Quebec: Comisión para la Cooperación Ambiental.

Ceballos, F. (2013). La vivienda Net Zero Energy. Memorias del Congreso Nacional de Vivienda. Eje Temático 5. Medio Ambiente y Vulnerabilidad. Ciudad de México: UNAM. 
Comisión Nacional de Vivienda (Conavi). (2008). Criterios e indicadores para los desarrollos habitacionales sustentables en México. Ciudad de México.

Comisión Nacional de Vivienda (Conavi). (2018). Reglas de operación del Programa de Acceso al Financiamiento para Soluciones Habitacionales 2018. Diario Oficial de la Federación, 23 de marzo. Ciudad de México.

Comisión Nacional de Vivienda y Secretaría del Medio Ambiente y Recursos Naturales (2012). NAMA apoyada para la vivienda sustentable en México. Acciones de mitigación y paquetes financieros. Ciudad de México: Conavi / Semarnat / GIZ.

Consejo Nacional de Evaluación de Política de Desarrollo Social (Coneval). (2018). Estudio diagnóstico del derecho a la vivienda digna y decorosa. Ciudad de México. https://www.coneval. org.mx/Evaluacion/IEPSM/Documents/Derechos_Sociales/ Estudio_Diag_Vivienda_2018.pdf

Cortés, L. (1995). La cuestión residencial. Bases para una sociología del habitar. Madrid: Fundamentos.

Cortés, F. y Rubalcava, R. M. (1987). Métodos estadísticos aplicados a la investigación en ciencias sociales. Análisis de asociación. Ciudad de México: El Colegio de México, A.C.

Deffis Caso, A. (1982). Página web personal del arquitecto Armando Deffis Caso. http://www.armandodeffis.com.mx/index.php (consulta: 12 de marzo de 2019).

INEGI (Instituto Nacional de Estadística y Geografía). (2010). Censo de Población y Vivienda 2010. Resultados definitivos. Aguascalientes, México. https://www.inegi.org.mx/programas/ ccpv/2010/

INEGI (Instituto Nacional de Estadística y Geografía). (2015). Encuesta Intercensal 2015. Aguascalientes. México. https://www. inegi.org.mx/programas/intercensal/2015/

INEGI (Instituto Nacional de Estadística y Geografía). (2018). Encuesta Nacional sobre Consumo de Energéticos en Viviendas Particulares (Encevi) 2018. Aguascalientes. México. https:// www.inegi.org.mx/programas/encevi/2018/

INEGYCEI (Inventario Nacional de Emisiones de Gases y Compuestos de Efecto Invernadero). (2014). Análisis de resultados. Ciu- 
dad de México. https://www.gob.mx/cms/uploads/attachment /file/312045/INEGYCEI6CN_26_marzo_2018.pdf

Infonavit (Instituto del Fondo Nacional de Vivienda para los Trabajadores). (2019). Resultados operativos de la Hipoteca Verde. http:// portal.infonavit.org. $\mathrm{mx} / \mathrm{wps} / \mathrm{wcm} /$ connect/infonavit/cuentas +claras+transparencia/Informacion_obligatoria_art70/XXX+ Estadisticas

Lezama, J. L. (2002). Teoría social, espacio y ciudad. Ciudad de México: El Colegio de México, A.C.

Lezama, J. L. y Domínguez, J. (2006). Medio ambiente y sustentabilidad urbana. Papeles de Población, 12(49), 153-176. http:// www.redalyc.org/pdf/112/11204906.pdf

Meadows, D. H., Meadows, D. L., Randers, J. y Behrens, W. (1972). Los límites del crecimiento: informe al Club de Roma sobre el predicamento de la humanidad. Ciudad de México: Fondo de Cultura Económica.

Morillón, D. (2009). Vivienda sustentable en México. Revista Mundo HVACR, ANFIR-ANDIRA, 8(104), 32-39. https://www.mundo hvacr.com.mx/2009/08/viviendas-sustentables-en-mexico/

Morillón, D. (2011). Edificación sustentable en México: retos y oportunidades. Ciudad de México: Academia de Ingeniería de México. http://www.ai.org.mx/ai/archivos/ingresos/morillon/trabajo_ final.pdf

Morillón, D. y Ceballos, F. (2017). Sustainable energy for houses, sustainable energy technologies. CRC Press Taylor / Francis Group.

Morillón, D. y Morillón, R. (1993). Bioclimática: sistemas pasivos de climatización. México: Universidad de Guadalajara.

Ortiz, A., Malagón, S. y Masera, O. (2015). Ecotecnología y sustentabilidad: una aproximación para el sur global. Interdisciplina, 3(7), 193-215. http:/www.revistas.unam.mx/index.php/inter/ article/view/52391

Ortiz, A., Masera, O. y Fuentes, A. (2014). La ecotecnología en México. Morelia: UNAM. http://ecotec.unam.mx/Ecotec/wp-content /uploads/La-Ecotecnolog--a-en-M--xico-ENE-2015-BR.pdf

ONU Hábitat. (2019). Por un mejor futuro urbano. Viviendas y mejoramiento de asentamientos precarios. https://onuhabitat.org. 
$\mathrm{mx} /$ index.php/viviendas-y-mejoramiento-de-asentamientosprecarios

Portillo, A. y Sirvent, G. (1987). Tecnologías alternativas para el desarrollo urbano. Ciudad de México: Centro de Eco Desarrollo. Rojas, M. N. (2015). Ponencia presentada el 17 de septiembre en el Seminario Otra mirada a los estudios sobre la ciudad, organizada por el Posgrado de Urbanismo, Ciudad de México, UNAM.

Schelesselman, J. (1982). Case-control studies. Design, conduct, analysis. Nueva York: Oxford University Press.

Secretaría de Energía (Sener). (2011). Indicadores de eficiencia energética en México: 5 sectores, 5 retos. Ciudad de México: Secretaría de Energía / Agencia Internacional de Energía. https://www. gob.mx/cms/uploads/attachment/file/85305/Bibliograf_a_6.pdf Sistema de Evaluación de la Vivienda Verde. (2014). Sisevive-Ecocasa. Sistema de Evaluación de la Vivienda Verde. Ciudad de México. https://www.gob.mx/cms/uploads/attachment/file/84276/SISE VIVECONUEE.pdf

Sistema de Información e Indicadores para la Vivienda. (2018). SNIIV-Conavi. Ciudad de México. https://sniiv.conavi.gob.mx/inicio/ acerca_de.aspx

Tudela, F. (1982). Ecodiseño. Ciudad de México: Universidad Autónoma Metropolitana, Xochimilco.

Uchida, H. (2005). Eco-Technology. Human environment conscious science and technology. En The Honda Foundation International Symposium, Hanoi linking innovation and entrepreneurship for developing countries (pp.189-195). Vietnam.

\section{Acerca de los autores}

Perla Yannelli Fernández Silva es doctora en Ciencias Sociales, con especialidad en Estudios Socioterritoriales, por la Universidad Autónoma Metropolitana, Xochimilco; es maestra en Ciencias en Recursos Naturales y Desarrollo Rural, por El Colegio de la Frontera Sur, y arquitecta por la Universidad Iberoamericana. Realizó una estancia posdoctoral en el Posgrado de Urbanismo de la Universidad Nacional Autónoma de México (UNAM). Actualmente es catedráti- 
ca en las maestrías de Ciencias de la Sostenibilidad, de la UNAM, y Arquitectura Sustentable, de la Universidad Cuauhtémoc de Querétaro. Es candidata al Sistema Nacional de Investigadores. Sus líneas de investigación son: vivienda y urbanismo sustentable, relación campo ciudad en los bordes urbanos, e impactos urbanos y sociales de los megaproyectos. Colaboró en la Comisión Nacional de Vivienda. Participó en el Proyecto de Evaluación del Impacto Social del Tren México-Toluca, del Instituto de Ingeniería, UNAM. ORCID: https://orcid.org/0000-0001-6867-8318

Entre sus publicaciones destacan:

Fernández, P., Suárez, M. y Quiroz, H. (coords.). (2018). La movilidad en la Ciudad de México. Impactos, conflictos y oportunidades. Ciudad de México: UNAM, Instituto de Geografía.

Fernández, P. y De la Vega, S. (2017). ¿Lo rural en lo urbano? Localidades periurbanas en la Zona Metropolitana del Valle de México. Eure, Revista Latinoamericana de Estudios Urbano Regionales, 130(43), 185-206. https://scielo.conicyt.cl/scielo. php?pid=S0250-71612017000300185\&script=sci_arttext\&tl $\mathrm{ng}=\mathrm{en}$

Fernández, P. y Morillón, D. (2016). Uso diferenciado de dispositivos con aprovechamiento de la energía renovable para la vivienda de la Ciudad de México. Revista de Docencia e Investigación Educativa, 2(5), 33-48. http://www.ecorfan.org/spain/research journals/Docencia_e_Investigacion_Educativa/vol2num5/ Revista_de_Docencia_e_Investigacion_Educativa_V2_N5_5. pdf

David Morillón Gálvez es doctor en Ingeniería por la Universidad Nacional Autónoma de México (UNAM); maestro en Diseño Bioclimático, por la Universidad de Colima; e ingeniero civil por la Universidad de Guadalajara. Sus líneas de investigación son: diseño bioclimático, sustentabilidad energética de edificios y ciudad, aprovechamiento de energías renovables, cambio climático, y procesos y uso eficiente de energía. Pertenece al Sistema Nacional de Investigadores, nivel II, y actualmente es investigador en el Instituto de Ingeniería de la UNAM. Colabora en el proyecto sobre el tren suburbano Toluca-Ciudad de México, "Nivel de sustentabilidad del proyecto 
obra de la SCT-2018". Participa en la Red Iberoamericana de Geotermia Somera del Programa Iberoamericano de Ciencia y Tecnología para el Desarrollo, 2018, y en el proyecto de Centro de Investigación en Energía del Océano, "Bioclima y consumo de energía en México". ORCID: https://orcid.org/0000-0002-9178-3092

Entre sus publicaciones destacan:

Torres-Rodríguez, A., Morillón-Gálvez, D., Aldama-Ávalos D., Morales-Ramírez, J. D. y García Kerdan, I. (2019). Predicting the thermal behaviour of sands considering its moisture content and grain size with applications to geothermal heat pump installations. Energy and Buildings, 194, 85-104. https://www.science direct.com/science/article/abs/pii/S037877881833620X

Rosas Flores, J., Zenón Olvera, E. y Morillón-Gálvez, D. (2019) Potential energy saving in urban and rural households of Mexico with solar photovoltaic systems using geographical information system. Renewable and Sustainable Energy Reviews, 116. https: //www.sciencedirect.com/science/article/abs/pii/S1364032 119306203

Torres-Rodríguez, A., Morillón-Gálvez, D., Aldama-Ávalos, D., Hernández-Gómez, V. H. y García Kerdan, I. (2020). Thermal performance evaluation of a passive building wall with CO2-filled transparent thermal insulation and paraffin-based PCM. Solar Energy, 205, 1-11. https://www.sciencedirect.com/science/ article/abs/pii/S0038092X20304758

Recepción: 4 de abril de 2019. Aceptación: 18 de junio de 2019. 
